Dermatologische Zeitschrift. 1912;19:I-V

\title{
Contents, Vol. 19, 1912
}

\section{Zeitschrift}

Begründet von O. LASSAR

Unter Mitwirkung von A. Alexander-Berlin; J. Almkvist-Stockholm; G. Arndt-Berlin; Ed. Arning-Hamburg; S. Bettmann-Heidelberg; B. Bloch-Basel; C. Bruhns-Berlin; A. BuschkeBerlin; J. Darier-paris; K. Dohi-Tokio; S. Ehrmann-Wien; J. Fabry-Dortmund; C. GrouvenHall $\beta$; J. Heller-Berlin; H. Hübner-Marburg: A. Jesionek-Giessen; F. Juliusberg-Posen; A. LanzMoskau; P. Linser-Tübingen; R. Matzenauer-Graz; G. Nobl-Wien; A. Pasini-Mailand; G.PernetLondon; F.Pinkus-Berlin; C.Rasch-Kopenhagen; E.Riecke-LOipzig; J. Schaeffer-Breslau; G. Scherber-Wien; H. E. Schmidt-Berlin; Frank Schultz-Berlin; 8. Spiethoff-Jena; C. SternDüsseldorf; E. Tomasczewski-Berlin; L. Török-Budapßst; H. Vörner-Leipzig; J. WertherDresden; Ch. J. White-Boston; A. Whitfield-London; J. Zeisler-Chicago; K. Zieler-Würzburg; F. Zinsser-Köln a. Rh. mid in Gemeinschaft mit

A. Blaschko K. Herxheimer V. Klingmüller M. Wolters

Herlin Frankfurt a. M. Kiel Rostock

Herausgegeben von

E. Hoffmann

Bonn.

Band XIX.

Mit z,ihirei $<\cdot$ hen Abbildungen im Text imd 20 Tafeln.

BERLIN 1912

YERLAG VON S. KARGER

KARLSTR $\Lambda$ SSE 15.

$\neq \mathrm{IV} \backslash$

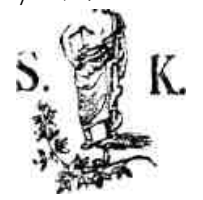

Alle Rechte vorbehalten.

Gedruokt bei Imberg \& Leíson G. m. b. H. in Berlin SW. 68.

Inhalts -Verzeichnis.

Seite

Original-Arbeiten.

Almkvist, J., Weitere Untersuchungen über die Patho-genese der merkuriellen Colitis und Stomatitis. (Hierzu

ïaf. XI bis XVIII) 949, 1057

Altmann, K., Die Serodiagnostik der Syphilis .... 22 A o k i , i., Zur Frage „, Tätowierung und Syphilis". (Hierzu

Taf. VII) $\quad .508$

Blumenthal, Franz, und Ludwig Hercz, Versuche zur Verschärfung der Wassermannschen 
Reaktion bei Syphilis 769

Brauer, A,, Zur Kenntnis der Salvarsandermatosen . . 800 Brückler, 0., Beitrag zur Kenntnis der fieberhaften

Reaktionen nach intravenösen Salvarsaninjektionen . . 127 F i m m e n, Lymphatische

Leukämie mit Hauttumoren . 705 Géber, Hans, Über das Wesen der sogenannten idiopathischen Erytheme 782

Heller, Julius, Zur Kasuistik seltener Nagelerkran-

kungen. X. Zur Pathogenese der Onycholysis . . 609 Heuck, Wilhelm, Über ,,Granuloma pediculatunv' (sog. menschliche Botryomykose). (Hierzu Taf. ПI

bis VI) 221, 324, 404

Hoffmann, E rich, Wer ist der ,,Pfälzer Anonymus iiC 1043 H ü b n e r , H., Über eine bisher nicht bekannte Neben-

wirkung des Yohimbins

863

Jordan, A., Über Versuche mit Neosalvarsan .... 992 Kudisch, W. M., Drei Fälle von

Bromoderma tuberosum vegetans aut papillomatosum. (Exanthemata ex usu

Bromi.) 713

L i e r, W., Die histologischen Veränderungen der Haut-

syphilide durch Salvarsan. (Hierzu Taf. II) .... 315

$-\mathrm{IV}-$

L·oeb, Felix, Beitrag zur Kenntnis der Röntgenver-

brennungen. (Hierzu Taf. I) 250

Meyer, L u d w i g, Zur endovenösen Therapie der Lues

mit Hg-Präparaten 393

$\mathrm{N}$ e u s t a d t , Max, Über Cancer en cuirasse mit Blasen-

bildung und Lokalisation an der Haut des rechten Ober-

schenkels und des Unterbauches 487

$\mathrm{P}$ a g e $\mathrm{n}$ s t e c h e r, Alexander, Behandlung von

syphilitischen Nasengeschwüren im Jahre 1820 . . 420 Pawlow, P. A., Resultate längerer

klinischer Beobach-

tungen über die Behandlung der Syphilis mit Salvarsan 149

P日ller, S., $\lambda$ rersuche der Detätowierung. . 900

Pinkus, Felix, Zur Kenntnis der • Himschwellungs-

erscheinungen während der Syphilis behandlung. . . . 675 R a s c h , C, Sklerodermie mit

Affektion der Mundschleim-

haut und Basedow-Addison-Symptomen. Bemerkungen

über die Ätiologie der Krankheit 244

R o h r b a c h, R., Über neuere Behandmngsmethoden

gonorrhoischer Komplikationen $\quad \ldots \quad 1$

Scheuer, O s k a r , Syphilidophobie 46

Schlasberg, H. J., Der Einfluß des Salvarsans auf die

Nieren bei intravenösen Injektionen 807

Schmidt, Willy, und F. Wagner, Beitrag zur

pathologischen Anatomie der Dermatitis papillaris capil-

litii (Kaposi), Folliculitis nuchae sclerotisans. (Hierzu

Taf. VIII-IX) 581

Turk, N o r b e r t , Zur Kenntnis der idiopathischen geni- 
talen Lymphangiektasie

138

Vignolo-Lutati, Karl, Beitrag zum Studium der

Sclerodermia circumscripta. (Hierzu Taf. X) .... 592 W a g n e r , F., Vorläufige Mitteilungen über die Anwendung

von Thorium X in der Dermatologie 988

Zinsser, F., und P. Philip p, Ulcus cruris varicosum

und Syphilis. (Hierzu Taf. XIX-XX) 1051

Gesellsehaftsberichte.

Bericht über den VIII. Kongreß der Deutschen Röntgen-

gesellschaft in Berlin, Langenbeckhaus, 13. und 14. April

1912. Von Dr. Arthur Alexander in Charlotten-

burg 436

84. Versammlung Deutscher Naturforscher und Ärzte zu

$\mathrm{V}-$

Münster i. W. vom 15.-21. September 1912. Bericht von Dr. Tecklenborg in Münster .... 1027, 1091

VII. Internationaler Dermatologen-Kongreß, 9.-13. April

1912421

Berliner Dermatologische Gesellschaft. Festsitzung zur Feier

des 25jährigen Bestehens am 28. Oktober 1911 ... 51

Ordentliche General versammlung am 14 . No

vember 1911166

-匹 - - Sitzung am 12. Dezember 1911, 9. Januar, 18. Februar, 13. März, 14. Mai, 11. Juni und 9. Juli 1912 . . 252,

$343,445,520,613,720,1078$

Krakauer medizinische Gesellschaft. Sitzung am 11. Oktober

$1911 \ldots$

183

Moskauer venerologisch-dermatologische Gesellschaft. Sit

zungen am 27. November, 10. Dezember 1911 und am

25. Februar und 15. April $1912 \quad 182,285,636$

Pariser Dermatologische Gesellschaft. Sitzung am 4. Januar.

1. Februar, 7. März, 4. April, 2. Mai, 6. Juni und

4. Juli $1912 \quad 283,359,629,739,918$

Proceedings of the Royal Society. Dermatologische Sektion.

Sitzungen im Jahre $1911 \cdot 277$

Proceedings of the Royal Society of Medicine. Sitzungen vom

Г4. Dezember 1911, 18. Januar, 15. Februar, 21. März,

18. April, 16. Mai $1912 \quad$.... 1022

Warschauer ärztlicher $\lambda \tau$ erein. Sitzung am 16. Mai $1911 \ldots 183$

Wiener Dermatologische Gesellschaft. Sitzung vom 22. November und 6. Dezember 1911, und vom 17. Januar. 7. Februar, 28. Februar, 13. März, 15. Mai, 29. Mai

und 12. Juni $1912 \quad 85,180,273,356,461$,

$626,737,816$

Periodische Literatur 88, 185, 286, 360, 466, 534, 638, 744,

$818,922,999,1095$

Buehanzeigen 122, 217, 311, 391, 484, 765, 1032 
Tagesnachrichten und Personalien 126, 314, 486, 674, 768, 862, 947, 1042, 1120

Dr. E. Heuß† 1042

Magnus Möller $\dagger 768$

Dr. J. Neuberger $\uparrow \quad 1041$

Sachregister 1121

Namenregister

1137 